OPEN ACCESS

Edited by:

Yue-Feng Liu,

Jilin University, China

Reviewed by:

Jianxun Dai,

Dalian University of Technology, China

Jing Cao,

Tianjin Polytechnic University, China

${ }^{*}$ Correspondence:

Ziying Wang

wangzy@hebut.edu.cn

Lingxiao Gao

lingxiao.gao@hebut.com

Specialty section:

This article was submitted to Nanoscience,

a section of the journal

Frontiers in Chemistry

Received: 08 November 2021 Accepted: 24 November 2021

Published: 24 December 2021

Citation:

Ma Z, Wang Z and Gao L (2021) LightAssisted Enhancement of Gas Sensing Property for Micro-Nanostructure Electronic Device: A Mini Review.

Front. Chem. 9:811074.

doi: $10.3389 /$ fchem.2021.811074

\section{Light-Assisted Enhancement of Gas Sensing Property for Micro-Nanostructure Electronic Device: A Mini Review}

\author{
Zongtao $\mathrm{Ma}^{1}$, Ziying Wang ${ }^{2,3 *}$ and Lingxiao $\mathrm{Gao}^{2,3 *}$ \\ ${ }^{1}$ State Key Laboratory of Reliability and Intelligence Electrical Equipment, Hebei University of Technology, Tianjin, China, ${ }^{2}$ National \\ Engineering Research Center for Technological Innovation Method and Tool, School of Mechanical Engineering, Hebei University \\ of Technology, Tianjin, China, ${ }^{3}$ School of Electronics and Information Engineering, Hebei University of Technology, Tianjin, China
}

In recent years, gas sensing electronic devices have always attracted wide attention in the field of environment, industry, aviation and others. In order to improve the gas sensing properties, many micro- and nano-fabrication technologies have been proposed and investigated to develop high-performance gas sensing devices. It is worth noting that light irradiation is an effective strategy to enhance gas sensitivity, shorten the response and recovery time, reduce operating temperature. In this review, firstly, the latest research advances of gas sensors based on different micro-nanostructure materials under UV light and visible light activation is introduced. Then, the gas sensing mechanism of light-assisted gas sensor is discussed in detail. Finally, this review describes the present application of gas sensors with improved properties under light activation assisted conditions and the perspective of their applications.

Keywords: light irradiation, micro-nanostructure, gas sensing devices, sensor, metal oxide semiconductor

\section{INTRODUCTION}

With the rapid improvement of economy and the quality of human life, people have realized that environmental pollution has caused irreparable damage to the Earth. Harmful gases from coal-fired power stations, garbage incineration, automobile exhaust and industrial waste gas not only pose a threat to the environment but also endanger human health (Cheng et al., 2021a). Therefore, the development of harmful gas detection technology is of great significance.

The metal oxide semiconductor (MOS) sensors have many advantages of small size, high response, fast response and so on, which attract great interest in real-time detection of different gases. However, the high operating temperature can reduce the life of the device and sensitivity. Consequently, researchers began to explore gas sensing devices by putting forward a large number of theoretical methods and experimental schemes, which can work at low temperature or even room temperature (RT). In recent years, with the continuous development of nanotechnology and nanomaterials, the research of RT gas sensor has also got significant progress include morphological control (Wang et al., 2021, noble metal surface modification (Wang et al., 2019a) or doping and the formation of heterostructures (Wang et al., 2019b). Furthermore, light activation is an effective method to improve the performance of MOS sensors. The optical irradiation of MOS sensor can change the surface electronic properties by adjusting the concentration of optical carriers in MOS, so as to promote the interaction between molecule and sensor layer (Kumar et al., 2020). Herein, we will summarize the latest progress of photoactivated RT MOS sensors in the past few years. 
A

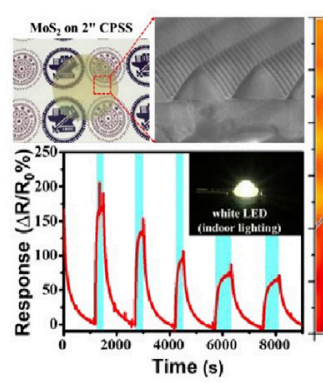

C

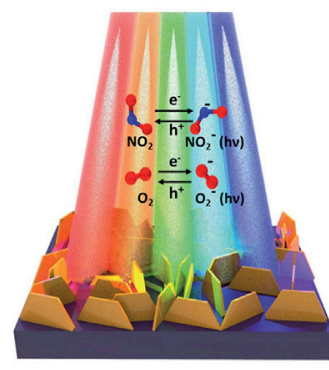

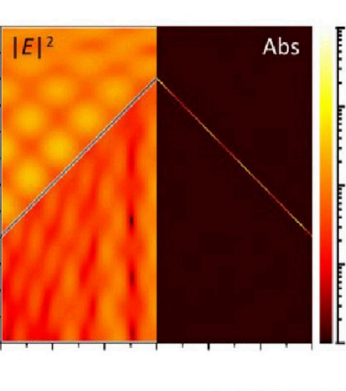
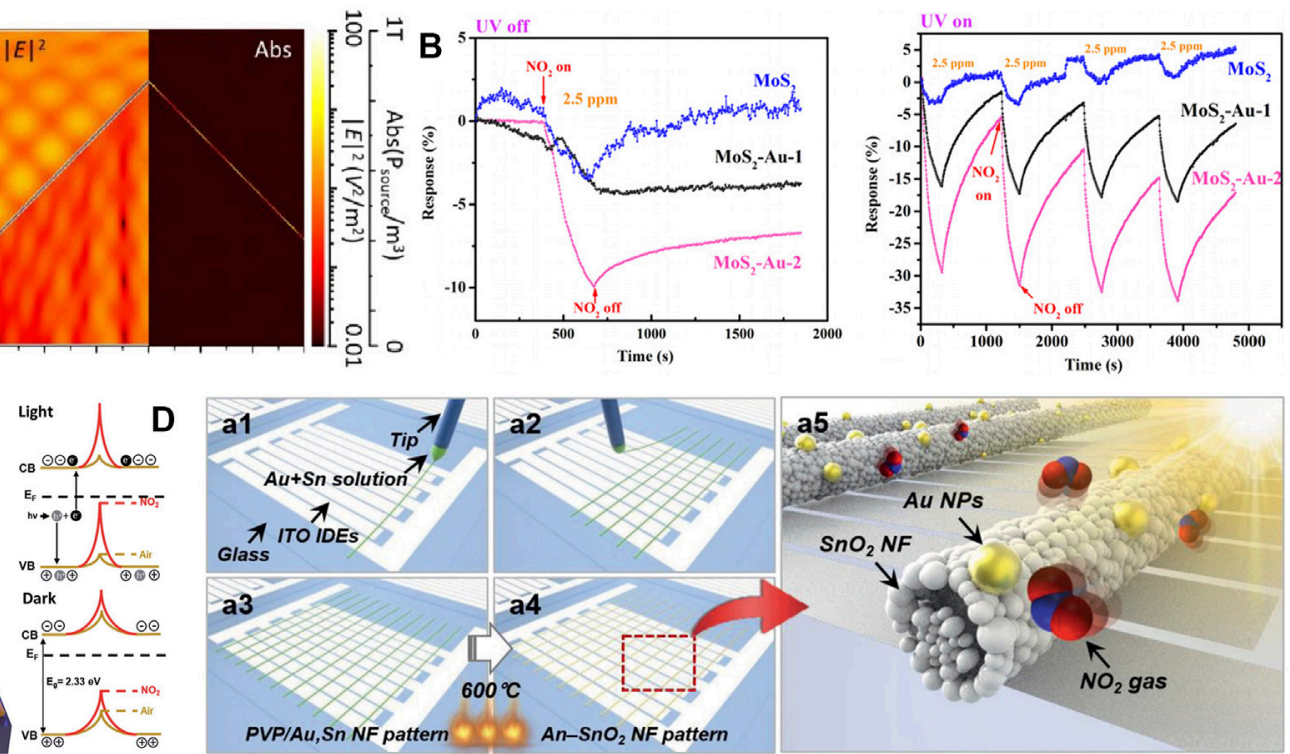

Time (s)

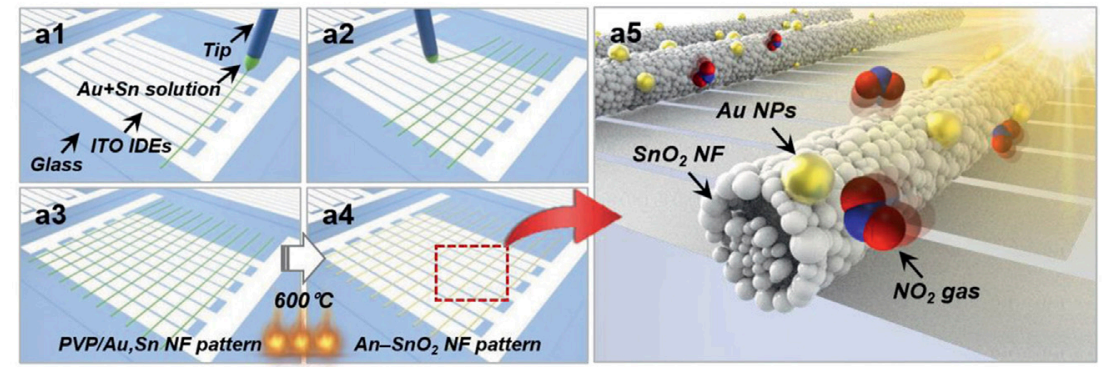

FIGURE 1 | (A) Microscopic characterization of $\mathrm{MoS}_{2}$ and gas sensing performance under white light, Simulation results for the Flat-MoS 2 and Cone-Shaped$\mathrm{MoS}_{2}$ gas sensors by finite difference time domain (FDTD) illuminated by the light with the wavelength of $550 \mathrm{~nm}$. Reproduced with permission from ref. 13 . Copyright 2019, Royal Society of Chemistry. (B) Schematic illustrations of edge-enriched $\mathrm{SnS}_{2} \mathrm{NFs}$ for visible light activated $\mathrm{NO}_{2}$ gas sensor. Reproduced with permission from ref. 14. Copyright 2021, Royal Society of Chemistry. (C) Sensing performance of $\mathrm{MoS}_{2}$ and $\mathrm{MoS}_{2}$-Au sensors toward $\mathrm{NO}_{2}$ gas with no UV illumination. Reproduced with permission from ref. 17. Copyright 2018, American Institute of Physics. (D) Schematic illustration for (left) the experimental procedures to fabricate nanofiber pattern (NF-P) sensors via near-field electrospinning (NFES) and (right) $\mathrm{NO}_{2}$ detection of nanofiber pattern (NF-P) sensors under visible light illumination. Reproduced with permission from ref. 18. Copyright 2021, WILEY-VCH.

Recently, many reports have confirmed that ultraviolet (UV) irradiation can indeed improve the performance of MOS sensors, including higher sensitivity, shorter recovery time and lower power consumption. UV excitation can increase the density of free electron-hole pairs and lead to photodissociation of the target gas and chemical surface adsorbents (Zhai et al., 2018; Park et al., 2014; Hyodo et al., 2017). However, the harmful effects of UV light on human skin and eyes remain an acute problem, and UV light accounts for only 5-7\% of the total energy from sunlight. Therefore, additional UV-LED light sources are required, which presents a new challenge to the size design of the sensor. By contrast, visible light is superior to UV light in terms of energy acquisition and energy utilization. More importantly, it does no harm to human health. Similarly, visible light activation of narrow band gap metal oxides for RT gas sensing has been explored by numerous researchers in the past few years (Hasani et al., 2015; Li et al., 2018; Song et al., 2020). While visible light activation is an effective way to improve the performance of MOS sensors, it still faces great challenges to widely apply it in real environment.

In this paper, we discuss RT gas sensors under light assisted conditions. This review is divided into three parts: firstly, we focus on the latest progress of gas sensors based on MOS, noble metal doped MOS and MOS with heterojunction structure at room temperature by different wavelength light sources (UV light and visible light). Secondly, we describe the gas sensing mechanism under light-assisted condition. Finally, we introduce the application status and future prospects of gas sensors under light-assisted conditions.

\section{LIGHT ACTIVATION GAS SENSORS BASED ON DIFFERENT MICRO-NANOSTRUCTURE MATERIALS}

Many studies have shown that light irradiation is one of the effective ways to improve the gas sensitivity of pure MOS, noble metal doping MOS nanostructure and MOS with heterostructure can be improved under the condition of light activation, which will be described one by one below.

\section{Gas Sensors Based on Pure Metal Oxide Nanostructure}

In 2008, Costello and his colleagues firstly confirmed that UV light can improve the RT sensitivity of $\mathrm{ZnO}$ sensor. The $\mathrm{ZnO}$ sensor could detect $1 \mathrm{ppb}$ of acetaldehyde and acetone and obtained adjustable sensitivity though manipulating the intensity of UV light (De Lacy Costello et al., 2008). Chen et al. demonstrated the gas sensor based 3D cone-shaped $\mathrm{MoS}_{2}$ bilayer showed high response ( 470\%) and short response time $(\sim 25 \mathrm{~s})$ after exposure to $1 \mathrm{ppm}$ of $\mathrm{NO}$ under UV light (Chen et al., 2019). As shown in Figure 1A, it is explained that the excellent NO sensing properties is due to 
the three-dimensional light scattering effect attracted by UV light, which further enhances the light absorption.

Because of the health hazards and low utilization of UV light, researchers began to explore the replacement of UV light with visible light. Li et al. prepared a highly ordered CdS nanoflakes array by using Chemical Vapor Deposition (CVD) technology and studied its gas sensing characteristics (Li et al., 2018). It is found that the sensor has good working potential under natural solar lamps and can be used for outdoor environment monitoring. The excellent gas performances are attributed to the low band gap energy $(2.4 \mathrm{eV})$ and the unique morphology of $\mathrm{CdS}$. These inherent properties of CdS can enhance light absorption and conductivity. Wang et al. successfully prepared $\mathrm{SnS}_{2}$ nanoflowers by solvothermal synthesis (Eom et al., 2021). In Figure 1B, it is confirmed that the high absorbance of $\mathrm{SnS}_{2}$ in the visible region triggered the generation of carriers, which could decrease the resistance and enhance the gas sensing characteristics. Friedman et al. fabricated $\mathrm{MoS}_{2}$ by mechanical stripping and tested the sensitivity of the sensor under visible light (Friedman et al., 2014). They observed a 10-fold difference in the sensitivity of the sensors to trimethylamine before and after light exposure. The improved gas sensitivity of $\mathrm{MoS}_{2}$ sensors comes from photoexcitation.

\section{Gas Sensors Based on Noble Metal Doping Nanostructure}

Many studies have demonstrated that the modification of noble metal and light irradiation have synergistic effect on improving the gas sensing performance of pure MOS. On one hand, Li et al. fabricated a sensor based on $\mathrm{Au} / \mathrm{ZnO}$ porous octahedron ( $\mathrm{POHs}$ ). Compared with the sensor based pure $\mathrm{ZnO} \mathrm{POH}$, the response of the sensor based on $\mathrm{Au} / \mathrm{ZnO} \mathrm{POHs}$ to formaldehyde is significantly improved under UV light (Tsai et al., 2018). Zhou et al. reported a UV-assisted, recoverable, highly sensitive and selective $\mathrm{NO}_{2}$ gas sensor based on $\mathrm{Au}-\mathrm{MoS}_{2}$ nanocomposite (Zhou et al., 2018a). In Figure 1C, the Au- $\mathrm{MoS}_{2}$ sensor has a three-fold enhanced response to $\mathrm{NO}_{2}$, full recovery property and good repeatability under UV light. The detection of $\mathrm{NO}_{2}$ under UV light provides an alternative strategy for the design of single multifunctional optoelectronic devices.

On the other hand, Kim et al. reported the detection of $\mathrm{NO}_{2}$ gas by a one-dimensional $\mathrm{Au}-\mathrm{SnO}_{2}$ nanofiber sensor under visible light at RT (Lim et al., 2021). The sensor shows a high degree of selectivity, sensitivity and repeatability in response to $\mathrm{NO}_{2}$ at sub ppm levels. As shown in Figure 1D, the excellent RT $\mathrm{NO}_{2}$ properties are in connection with the effects of $\mathrm{Au}$ nanoparticles from local surface plasmon resonance (LSPR) in visible light. This work makes the new transparent design possible for oxide gas sensors without external heaters or light sources. Additionally, Chen et al. developed a high-performance visible light activated $\mathrm{NO}_{2}$ gas sensor based on LSPR and increased surface oxygen vacancy (Chen et al., 2020). The results show that $\mathrm{Au}$ NPs modification can significantly improve the visible photosensitivity of $\mathrm{ZnO}$ films compared with pure $\mathrm{ZnO}$ films. It provides an effective way to construct high-performance photoactivated gas sensor.

\section{Gas Sensors Based on Heterojunction Nanostructure Composite}

Both the construction of heterostructure and light irradiation can increase active sites and charge transfer of pure metal oxide semiconductor. The heterostructure of MOS can enhance charge transduction and adjust grain boundary potential barrier, which is beneficial to improve the gas sensitivity. For one thing, Chang et al. prepared hollow $\mathrm{ZnO} / \mathrm{MoS}_{2}$ nanosheets with core/shell heterostructure (Chang et al., 2020). The high acetone response and fast response/recovery rate are caused by the fast gas transport channel and the n-p heterojunction of $\mathrm{MoS}_{2}$ nanosheets. Moreover, UV light is introduced to further improve the acetone reaction and greatly reduce the operating temperature. The optical diffraction and reflection caused by the decoration of layered $\mathrm{MoS}_{2}$ nanosheets can significantly improve the light capture. Yang et al. successfully synthesized hollow $\mathrm{ZnO}$ $\mathrm{SnO}_{2}$ heterostructure for triethylamine (TEA) (Yang et al., 2018). Particularly, the sensor based $\mathrm{ZnO}-\mathrm{SnO}_{2}$ showed short response time (1.8 s) and recovery time (18 s) under UV light.

For another, Liu et al. reported hollow $\mathrm{SnO}_{2} @ \mathrm{SnS}_{2}$ nanostructures prepared by one-step hydrothermal method (Liu et al., 2020). The sensor based on $\mathrm{SnO}_{2} @ \mathrm{SnS}_{2}$ has a fast response rate and good recovery ability to ppb-level $\mathrm{NO}_{2}$ under visible light at RT. The excellent sensing performance of $\mathrm{SnO}_{2} @$ $\mathrm{SnS}_{2}$ sensor is due to the hollow and porous heterojunction structure, which is conducive to gas diffusion, especially visible light assisted to promote charge transfer and gas desorption. Chen et al. prepared gas-sensitive $\mathrm{ZnO}$ nanorods with sea urchinlevel mesoporous structure modified by $\mathrm{PbS}$ quantum dots (QDs) (Chen et al., 2018). The PbS QDs have a narrow band gap shown in the Near Infrared Spectroscopy Analysis (NIR) spectrum. Compared with the original $\mathrm{ZnO}$ nanorods, the sensor based $\mathrm{ZnO} / \mathrm{PbS}$ nanocomposites have a higher response and faster response/recovery speed to ppm level $\mathrm{NO}_{2}$ under visible light.

\section{SENSING MECHANISM OF LIGHT-ASSISTED GAS SENSOR}

Light activation has been proved to be an effective way to improve the performance of gas sensors, but the relationship between the types of light source, the structure of materials and the improvement of gas sensing properties are not clear. The explanations for the improved gas sensitivity of UV light are as follows:

Firstly, Gong et al. proposed the gas sensor based on a novel $\mathrm{ZnO}$ hybrid with nanowire/optical fiber (Gong et al., 2017). The results show that UV irradiation can improve the sensitivity and shorten the response time, and the sensor also has good longterm stability. UV irradiation can respond to gas with ppb-level concentration at low temperature. The electrons in the $\mathrm{ZnO}$ hybrid excited by UV irradiation promote the reduction of ethanol gas, leading to the higher gas performance. Therefore, the irradiation of UV will generate more conductive electrons, improve the conductivity of the sensing materials and promote the electron transfer under UV irradiation, thus improving the 
response of the gas sensor. Secondly, Zhou et al. have proposed that the increased sensitivity is ascribed to the increase in the number of active adsorption sites and the introduction of active catalysts such as $\mathrm{Au}, \mathrm{Ag}$, Pd and other noble metals (Zhou et al., 2018a). For example, Au nanoparticles can accelerate the capture of more photons, resulting in additional photoexcited carriers to promote the interaction between gas and sensing materials. The efficient separation of photoexcited carriers at the interface of $\mathrm{MoS}_{2} / \mathrm{Au}$ also contributes to rapid and complete recovery under UV light. Finally, Zhou et al. explained that UV light can excite electron-hole pairs in $\mathrm{ZnO}$ (n-type) and $\mathrm{MoS}_{2}$ (p-type) (Zhou et al., 2018b). The photoexcited electrons in the conduction band can be easily transferred to $\mathrm{ZnO}$ by the built-in field, which is similar to the excited hole in the valence band of $\mathrm{ZnO}$ to the excited hole of $\mathrm{MoS}_{2}$. Therefore, light carriers can be effectively separated at the interface of heterojunction in order to improve the gas sensing properties.

The explanations for the improved gas sensitivity of visible light are as follows: Primarily, the explanations given by Li et al. are as follows: The photon energy of visible light greater than the band gap of semiconductor can produce electron-hole pairs. Electrons and holes cannot recombine efficiently, and the electrons of light source move to the conduction band and then are accumulated in the conduction band. This results in the high concentration of electrons in the conduction band and the high conductivity of the semiconductor. Therefore, the sensitivity of the gas sensor is significantly increased under visible light (Li et al., 2018). Posteriorly, Chen et al. developed a high-performance visible light activated $\mathrm{NO}_{2}$ sensor based on LSPR and increased surface oxygen vacancy (Chen et al., 2020). The mechanism of visible light activated by LSPR absorption are illustrated. At the interface of metal and semiconductor, the Fermi level of $\mathrm{ZnO}$ is transferred to a positive charge when electrons transfer from $\mathrm{ZnO}$ nanorods to Au nanorods, resulting in an upward-bending band interface and a Schottky barrier. The photoexcited electrons generated in $\mathrm{Au}$ can overcome the blocking and injection of conduction band in $\mathrm{ZnO}$, making more free electrons can participate in the chemical reaction with the surface adsorbed $\mathrm{O}_{2}$ and $\mathrm{NO}_{2}$ molecules. Niu et al. prepared vertically stacked $\mathrm{MoS}_{2} / \mathrm{GaSe}$ heterojunction by all-dry transfer method (Niu et al., 2021). When the p-n junction between $\mathrm{MoS}_{2}$ and GaSe is exposed to visible light, electronhole pairs in both $\mathrm{MoS}_{2}$ and GaSe layers are generated by light and tend to migrate at the interface. Due to the built-in electric field, electrons transfer to n-type $\mathrm{MoS}_{2}$ and holes transfer to p-type GaSe, resulting in carrier separation. $\mathrm{NO}_{2}$ has a strong electron affinity and can easily capture electrons from the conduction band of the material. In general, the separation of the light source carrier and the built-in electric field acts as a driving force to drive the gas sensing behavior.

The possible mechanism and reasons for the superior gas performance of light irradiation can be explained as follows:

1) For pure metal oxide nanostructure, the gas sensing performance depends on the amount of oxygen active substance adsorbed on the nanostructure surface. Under dark conditions, the concentration of free carriers inherent in the nanostructure is low, resulting in less utilization of the active site to produce absorbed oxygen. In contrast, with the increase of the number of free carriers under light irradiation, the surface absorption site improves the chemical activity of the surface and thus enhances the gas sensitivity.

2) For noble metal doping nanostructure, the LSPR effect not only enhances the absorption of light, but also inhibits the reorganization of electron-hole pairs produced by light. The intermediate-thermal electrons generated by LSPR absorption can overcome the Schottky barrier at the noble metal/oxide junction and inject into the oxide conduction band. Therefore, more surface adsorbed oxygen is formed on the oxide surface, leading to a stronger sensing reaction.

3) For heterojunction nanostructure composite, the formation of heterojunction can promote the separation of electron-hole pairs, effectively accelerate the electron conversion between different particles, and improve the response rate. When the heterojunction is exposed to light irradiation, electron-hole pairs in the semiconductor are generated by light and tend to migrate at the interface. Due to the built-in electric field, the electron transfer rate is enhanced, resulting in carrier separation and improving the gas sensitivity.

\section{THE PRESENT APPLICATION OF GAS SENSORS UNDER LIGHT ACTIVATION}

As essential for environmental monitoring, process control and early diagnosis of human disease, gas sensors under light activation are used to sense volatile organic compounds (VOCs). Wu et al. demonstrated a specific and highly sensitive detection of ketones using two-dimensional (2D) $\mathrm{MoTe}_{2}$ (Wu et al., 2018). They also investigated the effect of UV activation on the sensing performance of VOCs. Activation of UV light results in high sensitivity, low detection limit ( $\sim 0.2 \mathrm{ppm})$ to acetone and high stability even in high humidity, which is crucial for early diagnosis of diabetes. Zhao et al. demonstrated a flexible, transparent and high-performance gas sensor based $\mathrm{In}_{2} \mathrm{Se}_{3}$ using a simple pulsed laser deposition (PLD) method (Zheng et al., 2017). The gas sensor can work under the activation of visible light and shows excellent performance in detecting acetylene gas. No significant degradation of sensing performance was observed even after 100 bending cycles. The $\mathrm{In}_{2} \mathrm{Se}_{3}$ sensor has an average transmittance of $64 \%$ in the visible light range $(400-800 \mathrm{~nm})$. In addition, they found that the sensitivity, response and recovery rate of the sensor based $\mathrm{In}_{2} \mathrm{Se}_{3}$ depend on the light intensity. The excellent performance of $\mathrm{In}_{2} \mathrm{Se}_{3}$ sensors on flexible substrates provides a brilliant future for practical applications in wearable optoelectronic systems. Cheng et al. also prepared $\mathrm{CuO}$ flower-like materials (FMs) by hydrothermal method, and then obtained Au@CuO FMs by in-situ reduction reaction (Cheng et al., 2021b). The performance of the sensor based Au@CuO FMs was evaluated by detecting volatile and toxic gases such as ethanol, isopropanol, methanol and formaldehyde under UV light. It was found that the sensor based Au@CuO FMs had a higher response (95.3) than the sensor based $\mathrm{CuO}$ FMs at a 
concentration of 1,000 ppm, while the sensor based $\mathrm{Au} @ \mathrm{CuO}$ FMs had a response of 174 under UV light. Therefore, $\mathrm{Au} @ \mathrm{CuO}$ FMs is a promising ethanol detection method. In summary, light irradiation as an effective means enables gas sensors to maintain better performance in environmental monitoring and human health protection.

\section{CONCLUSION}

In this paper, we first introduce the latest research progress of gas sensors with different microstructures (pure metal oxide nanostructure, noble metal doping nanostructure, heterojunction composite nanostructure) activated by UV and visible light. In the next place, we introduce the light activation mechanism of gas sensors with different structures from the perspective of microscopic mechanism. Finally, several

\section{REFERENCES}

Chang, X., Qiao, X., Li, K., Wang, P., Xiong, Y., Li, X., et al. (2020). UV Assisted Ppb-Level Acetone Detection Based on Hollow ZnO/MoS2 Nanosheets Core/ shell Heterostructures at Low Temperature. Sensors Actuators B: Chem. 317, 128208. doi:10.1016/j.snb.2020.128208

Chen, C., Zhang, Q., Xie, G., Yao, M., Pan, H., Du, H., et al. (2020). Enhancing Visible Light-Activated NO2 Sensing Properties of Au NPs Decorated ZnO Nanorods by Localized Surface Plasmon Resonance and Oxygen Vacancies. Mater. Res. Express 7, 015924. doi:10.1088/2053-1591/ab6b64

Chen, R., Wang, J., Xia, Y., and Xiang, L. (2018). Near Infrared Light Enhanced Room-Temperature NO2 Gas Sensing by Hierarchical ZnO Nanorods Functionalized with PbS Quantum Dots. Sensors Actuators B: Chem. 255, 2538-2545. doi:10.1016/j.snb.2017.09.059

Chen, Y.-Z., Wang, S.-W., Yang, C.-C., Chung, C.-H., Wang, Y.-C., Huang Chen, S.-W., et al. (2019). An Indoor Light-Activated 3D Cone-Shaped MoS2 BilayerBased NO Gas Sensor with PPb-Level Detection at Room-Temperature. Nanoscale 11, 10410-10419. doi:10.1039/c8nr10157d

Cheng, M., Li, W., Li, C., Wang, Q., Tan, Q., Yang, W., et al. (2021a). Photochemical Sensitive Study of Au@CuO Flower-like Materials. Sensors Actuators B: Chem. 348, 130644. doi:10.1016/j.snb.2021.130644

Cheng, Y., Ren, B., Xu, K., Jeerapan, I., Chen, H., Li, Z., et al. (2021b). Recent Progress in Intrinsic and Stimulated Room-Temperature Gas Sensors Enabled by Low-Dimensional Materials. J. Mater. Chem. C 9, 3026-3051. doi:10.1039/ d0tc04196c

De Lacy Costello, B. P. J., Ewen, R. J., Ratcliffe, N. M., and Richards, M. (2008). Highly Sensitive Room Temperature Sensors Based on the UV-LED Activation of Zinc Oxide Nanoparticles. Sensors Actuators B: Chem. 134, 945-952. doi:10.1016/j.snb.2008.06.055

Eom, T. H., Cho, S. H., Suh, J. M., Kim, T., Lee, T. H., Jun, S. E., et al. (2021). Substantially Improved Room Temperature NO2 Sensing in 2-dimensional SnS2 Nanoflowers Enabled by Visible Light Illumination. J. Mater. Chem. A. 9, 11168-11178. doi:10.1039/d1ta00953b

Friedman, A. L., Keith Perkins, F., Cobas, E., Jernigan, G. G., Campbell, P. M., Hanbicki, A. T., et al. (2014). Chemical Vapor Sensing of Two-Dimensional MoS2 Field Effect Transistor Devices. Solid-State Electro. 101, 2-7. doi:10.1016/ j.sse.2014.06.013

Gong, B., Shi, T., Zhu, W., Liao, G., Li, X., Huang, J., et al. (2017). UV IrradiationAssisted Ethanol Detection Operated by the Gas Sensor Based on ZnONanowires/optical Fiber Hybrid Structure. Sensors Actuators B: Chem 245, 821-827. doi:10.1016/j.snb.2017.01.187

Hasani, A., Sharifi Dehsari, H., Amiri Zarandi, A., Salehi, A., Taromi, F. A., and Kazeroni, H. (2015). Visible Light-Assisted Photoreduction of Graphene Oxide Using CdS Nanoparticles and Gas Sensing Properties. J. Nanomater. 2015, 1-11. doi:10.1155/2015/930306 applications of photoactivated gas sensors in environmental monitoring, process control and early diagnosis of human diseases are listed.

\section{AUTHOR CONTRIBUTIONS}

All authors listed have made a substantial, direct and intellectual contribution to the work, and approved it for publication.

\section{FUNDING}

This research work was financially supported by Natural Science Foundation of Hebei Province (F2020202050) and the National Natural Science Foundation of China (Grant No. 52104189).

Hyodo, T., Urata, K., Kamada, K., Ueda, T., and Shimizu, Y. (2017). Semiconductor-type SnO2-Based NO2 Sensors Operated at Room Temperature under UV-Light Irradiation. Sensors Actuators B: Chem. 253, 630-640. doi:10.1016/j.snb.2017.06.155

Kumar, R., Liu, X., Zhang, J., and Kumar, M. (2020). Room-temperature Gas Sensors under Photoactivation: from Metal Oxides to 2D Materials. Nanomicro Lett. 12, 164. doi:10.1007/s40820-020-00503-4

Li, H.-Y., Yoon, J.-W., Lee, C.-S., Lim, K., Yoon, J.-W., and Lee, J.-H. (2018). Visible Light Assisted NO2 Sensing at Room Temperature by CdS Nanoflake Array. Sensors Actuators B: Chem. 255, 2963-2970. doi:10.1016/ j.snb.2017.09.118

Lim, K., Jo, Y. M., Yoon, J. W., Kim, J. S., Lee, D. J., Moon, Y. K., et al. (2021). A Transparent Nanopatterned Chemiresistor: Visible-Light Plasmonic Sensor for Trace-Level NO 2 Detection at Room Temperature. Small 17, 2100438. doi:10.1002/smll.202100438

Liu, D., Tang, Z., and Zhang, Z. (2020). Visible Light Assisted RoomTemperature NO2 Gas Sensor Based on Hollow SnO2@SnS2 Nanostructures. Sensors Actuators B: Chem. 324, 128754. doi:10.1016/ j.snb.2020.128754

Niu, Y., Zeng, J., Liu, X., Li, J., Wang, Q., Li, H., et al. (2021). A Photovoltaic SelfPowered Gas Sensor Based on All-Dry Transferred MoS 2 /GaSe Heterojunction for ppb-Level NO 2 Sensing at Room Temperature. Adv. Sci. 8, 2100472. doi:10.1002/advs.202100472

Park, S., Hong, T., Jung, J., and Lee, C. (2014). Room Temperature Hydrogen Sensing of Multiple Networked ZnO/WO3 Core-Shell Nanowire Sensors under UV Illumination. Curr. Appl. Phys. 14, 1171-1175. doi:10.1016/ j.cap.2014.06.019

Song, Y., Zhang, Y., Ma, M., Ren, J., Liu, C., and Tan, J. (2020). Visible LightAssisted Formaldehyde Sensor Based on HoFeO3 Nanoparticles with Sub-ppm Detection Limit. Ceramics Int. 46, 16337-16344. doi:10.1016/ j.ceramint.2020.03.191

Tsai, Y.-T., Chang, S.-J., Ji, L.-W., Hsiao, Y.-J., Tang, I.-T., Lu, H.-Y., et al. (2018). High Sensitivity of NO Gas Sensors Based on Novel Ag-Doped ZnO Nanoflowers Enhanced with a UV Light-Emitting Diode. ACS Omega 3, 13798-13807. doi:10.1021/acsomega.8b01882

Wang, Z., Gao, S., Fei, T., Liu, S., and Zhang, T. (2019b). Construction of ZnO/ $\mathrm{SnO} 2$ Heterostructure on Reduced Graphene Oxide for Enhanced Nitrogen Dioxide Sensitive Performances at Room Temperature. ACS Sens. 4, 2048-2057. doi:10.1021/acssensors.9b00648

Wang, Z., Sackmann, A., Gao, S., Weimar, U., Lu, G., Liu, S., et al. (2019a). Study on Highly Selective Sensing Behavior of Ppb-Level Oxidizing Gas Sensors Based on Zn2SnO4 Nanoparticles Immobilized on Reduced Graphene Oxide under Humidity Conditions. Sensors Actuators B: Chem. 285, 590-600. doi:10.1016/j.snb.2019.01.109

Wang, Z., Sun, J., Huo, Y., Yan, Y., Ma, Z., Bu, M., et al. (2021). Porous Co3O4 Nanocrystals Derived by Metal-Organic Frameworks on Reduced Graphene 
Oxide for Efficient Room-Temperature NO2 Sensing Properties. J. Alloys Comp. 856, 158199. doi:10.1016/j.jallcom.2020.158199

Wu, E., Xie, Y., Yuan, B., Hao, D., An, C., Zhang, H., et al. (2018). Specific and Highly Sensitive Detection of Ketone Compounds Based on P-type MoTe2 under Ultraviolet Illumination. ACS Appl. Mater. Inter. 10, 35664-35669. doi:10.1021/acsami.8b14142

Yang, T., Gu, K., Zhu, M., Lu, Q., Zhai, C., Zhao, Q., et al. (2019). ZnO-SnO2 Heterojunction Nanobelts: Synthesis and Ultraviolet Light Irradiation to Improve the Triethylamine Sensing Properties. Sensors Actuators B: Chem. 279, 410-417. doi:10.1016/j.snb.2018.10.031

Zhai, J., Wang, T., Wang, C., and Liu, D. (2018). UV-light-assisted Ethanol Sensing Characteristics of G-C3N4/ZnO Composites at Room Temperature. Appl. Surf. Sci. 441026, 317-323. doi:10.1016/j.apsusc.2018.02.026

Zheng, Z., Yao, J., Wang, B., and Yang, G. (2017). A Flexible, Transparent and High-Performance Gas Sensor Based on Layer-Materials for Wearable Technology. Nanotechnology 28, 415501. doi:10.1088/1361$6528 /$ aa 8317

Zhou, Y., Gao, C., and Guo, Y. (2018b). UV Assisted Ultrasensitive Trace NO2gas Sensing Based on Few-Layer MoS2nanosheet-ZnO Nanowire Heterojunctions at Room Temperature. J. Mater. Chem. A. 6, 10286-10296. doi:10.1039/ C8TA02679C
Zhou, Y., Zou, C., Lin, X., and Guo, Y. (2018a). UV Light Activated NO2gas Sensing Based on Au Nanoparticles Decorated Few-Layer MoS2thin Film at Room Temperature. Appl. Phys. Lett. 113, 082103-082107. doi:10.1063/1.5042061

Conflict of Interest: The authors declare that the research was conducted in the absence of any commercial or financial relationships that could be construed as a potential conflict of interest.

Publisher's Note: All claims expressed in this article are solely those of the authors and do not necessarily represent those of their affiliated organizations, or those of the publisher, the editors and the reviewers. Any product that may be evaluated in this article, or claim that may be made by its manufacturer, is not guaranteed or endorsed by the publisher.

Copyright (c) $2021 \mathrm{Ma}$, Wang and Gao. This is an open-access article distributed under the terms of the Creative Commons Attribution License (CC BY). The use, distribution or reproduction in other forums is permitted, provided the original author(s) and the copyright owner(s) are credited and that the original publication in this journal is cited, in accordance with accepted academic practice. No use, distribution or reproduction is permitted which does not comply with these terms. 